OPEN ACCESS

Edited by:

Karim Si-Tayeb,

INSERM U1087 L'unité de recherche de l'institut du thorax, France

Reviewed by:

Siegfried Hapfelmeier, University of Bern, Switzerland

Eva Rath,

Technical University of

Munich, Germany

*Correspondence:

Jorge O. Múnera

munera@musc.edu

Specialty section

This article was submitted to

Translational Medicine,

a section of the journal

Frontiers in Medicine

Received: 15 August 2019 Accepted: 29 November 2019 Published: 17 December 2019

Citation:

Daoud A and Múnera JO (2019) Insights Into Human Development and

Disease From Human Pluripotent

Stem Cell Derived Intestinal Organoids. Front. Med. 6:297.

doi: 10.3389/fmed.2019.00297

\section{Insights Into Human Development and Disease From Human Pluripotent Stem Cell Derived Intestinal Organoids}

\author{
Abdelkader Daoud and Jorge O. Múnera* \\ Department of Regenerative Medicine and Cell Biology, Medical University of South Carolina, Charleston, SC, United States
}

In recent years, advances in human pluripotent stem cell (hPSC) biology have enabled the generation of gastrointestinal (Gl) organoids which recapitulate aspects of normal organ development. HPSC derived gastrointestinal organoids are comprised of epithelium and mesenchyme and have a remarkable ability to self-organize and recapitulate early stages of human intestinal development. Furthermore, hPSC derived organoids can be transplanted into immunocompromised mice which allows further maturation of both the epithelium and mesenchyme. In this review, we will briefly summarize work from model systems which has elucidated mechanisms of Gl patterning and how these insights have been used to guide the differentiation of hPSCs into organoids resembling small intestine and colon. We will succinctly discuss how developmental principles have been used to promote maturation of human intestinal organoids (HIOs) in vitro as well as to introduce an enteric nervous system into HIOs. We will then concisely review how organoids have been used to study human pathogens, how new genetic and bioengineering tools are being applied to organoid research, and how this integration has allowed researchers to elucidate mechanisms of human development and disease. Finally, we will briefly discuss remaining challenges in the field and how they can be addressed. HPSC derived organoids are promising new model systems which hold the potential of unlocking unknown mechanisms of human gastrointestinal development and disease.

Keywords: human pluripotent stem cells, organoids, endoderm, midgut, hindgut

\section{GENERATION OF INTESTINAL ORGANOIDS}

In the past decade, organoid models have revolutionized the study of the gastrointestinal (GI) epithelium (1-4). Organoid models have provided significant insights into homeostasis and pathogenesis of GI organs. The most commonly used organoid models are derived from patient tissue, are grown as strictly epithelial structures and it has been suggested that they should be referred to as enteroids and colonoids (5). In addition, since they are derived from patients, they will reflect the age and physiology of the patient. Furthermore, enteroids and colonoids will reflect the region of intestine from which they are derived $(4,6)$. In contrast, human pluripotent stem cells (embryonic stem cells or induced pluripotent stem cells) can be differentiated into human intestinal and colonic organoids which contain both epithelial and mesenchymal cell types and reflect early stages of human gut development $(7,8)$. 
Studies in model organisms such as frog, mouse and chick are the foundation for directing human pluripotent stem cells toward desired tissue fates (9). Human intestinal organoids are generated by a stepwise differentiation which involves a series of growth factor manipulations which mimic mammalian development (Figure 1). First, definitive endoderm (DE) is specified using the NODAL mimetic Activin A (1012). DE induction is not a uniform process so co-developing mesodermal cells are also present in DE monolayers. This developing endoderm/mesoderm can then be patterned into mid/hindgut tissue through activation of the WNT and fibroblast growth factor (FGF) pathways (8). In addition to patterning the endoderm into caudal type homeobox 2 (CDX2) positive $\mathrm{mid} /$ hindgut tissue, WNT and FGF also induce 3-dimensional (3D) morphogenesis of endoderm and codeveloping mesoderm resulting in the formation of $\mathrm{mid} / \mathrm{hindgut}$ spheroids which bud off from the endoderm monolayer. These spheroids can then be grown in $3 \mathrm{D}$ culture using conditions developed in the Clevers lab $(4,13)$. This allows the development of human intestinal organoids (HIOs) which undergo morphological transitions similar to what occurs in normal mammalian development. HIOs also generate all the main epithelial and mesenchymal cells types present in the human intestine.

The generation of HIOs from hPSCs can be difficult to reproduce without proper training and experience. Suboptimal seeding density of hPSCs can result in the generation of definitive endoderm incapable of undergoing 3D morphogenesis into mid/hindgut spheroids. To circumvent this, several groups have generated HIOs by simply passaging WNT and FGF treated DE monolayers as clumps (14-17). When grown in 3D culture, these clumps can develop into HIOs which resemble HIOs generated from mid/hindgut spheroids. These studies indicate that patterned DE retains the capacity to self-organize even in the absence of spheroid morphogenesis. In addition, these studies provide an easier alternative to generating HIOs from spheroids.

HIOs have been utilized to study the effects of commensal bacteria on HIO physiology. Hill et al. utilized HIOs to investigate changes in the gene expression within immature human intestinal tissue upon the introduction of commensal Escherichia coli (E. coli) (18). Bacterial injection into the HIO lumen led to transcriptional changes that are normally associated with innate immune responses, and the activation of the NF-kB pathway. Using a fiber-optic optode to measure luminal oxygen levels in HIOs, the authors demonstrated that the transcriptional changes in HIOs injected with commensal bacteria were partially attributed to microbe-induced hypoxia. As a result, the naïve tissue exhibited an enhanced antibacterial response including increased mucus secretion, increased expression of beta defensins and elevated expression of tight junction proteins following injection of bacteria. This study indicates that commensal microbes can induce a complex physiological response in immature human intestinal tissue similar to responses seen in germ free mice that are colonized with commensal microbes.

\section{INTRODUCING COMPLEXITY AND MATURATION OF HIOS}

The co-development of mesoderm within HIOs suggested that there is a signaling crosstalk between the epithelium and the mesenchyme. Subsequent studies demonstrated that HIOs could be grown in the absence of NOGGIN and R-spondin indicating that the HIO mesenchyme is sufficient to maintain organoid growth in the absence of these and other factors, similar to what occurs in vivo $(19,20)$. This led researchers to hypothesize that co-developing mesoderm could support growth of HIOs following transplantation into the kidney capsule of immunocompromised mice. Following transplantation, HIOs matured into tissue that closely resembles human intestine with crypts, villi and all the major epithelial cell types present in the human intestine (19). In addition, the mesoderm from organoids formed circular and longitudinal smooth muscle layers. Engrafted tissue had a functional epithelial barrier, expressed functional brush border enzymes, had functional peptide absorption and had increased crypt fission following ileocecal resection (ICR) of the host. These studies highlight the utility of HIOs to model human gut physiology in vivo.

The smooth muscle layers of the intestine are innervated by the enteric nervous system which allows for peristalsis. The development of the smooth muscle layers in transplanted HIOs suggested that HIOs could be innervated (19). The enteric nervous system develops from vagal neural crest cells (NCCs). In order to establish an enteric nervous system in intestinal organoids, HIOs were mixed with vagal NCCs which were also derived from human pluripotent stem cells (21). In vitro, NCCs co-cultured with HIOs differentiated into distinct cell types of the human enteric nervous system (ENS) including neurons and glial cells. NCC differentiation and organization into ENS was supported by the HIOs which expressed the chemoattractant factors glial cell derived neurotrophic factor (GDNF) and endothelin 3 (EDN3). The resulting neurons were solely excitatory and displayed a spontaneous calcium efflux indicating a neuronal activity. The complexity of the neuronal system was further enhanced after in vivo transplantation resulting in the emergence of inhibitory neurons in addition to the excitatory neurons.

To functionally test the ability of HIOs with an ENS to produce peristalsis, electrical stimulation was applied to transplanted HIOs ex vivo. Upon receiving a neuronal stimulus, HIOs with an ENS exhibited contractile waves similar to peristalsis in human intestines suggesting a neuromuscular communication. Additionally, the NCC derived inhibitory neurons integrated into the smooth muscle layers to control tissue relaxation by releasing the neurotransmitter nitric oxide (NO). Furthermore, the presence of ENS following transplantation of the HIOs resulted in decreased expression of markers of differentiated epithelial cell types and increased epithelial proliferation in the HIOs. The generation of innervated intestinal tissue is a significant advancement since it could allow for the replacement of damaged intestine with a tissue which is competent to induce peristalsis. 


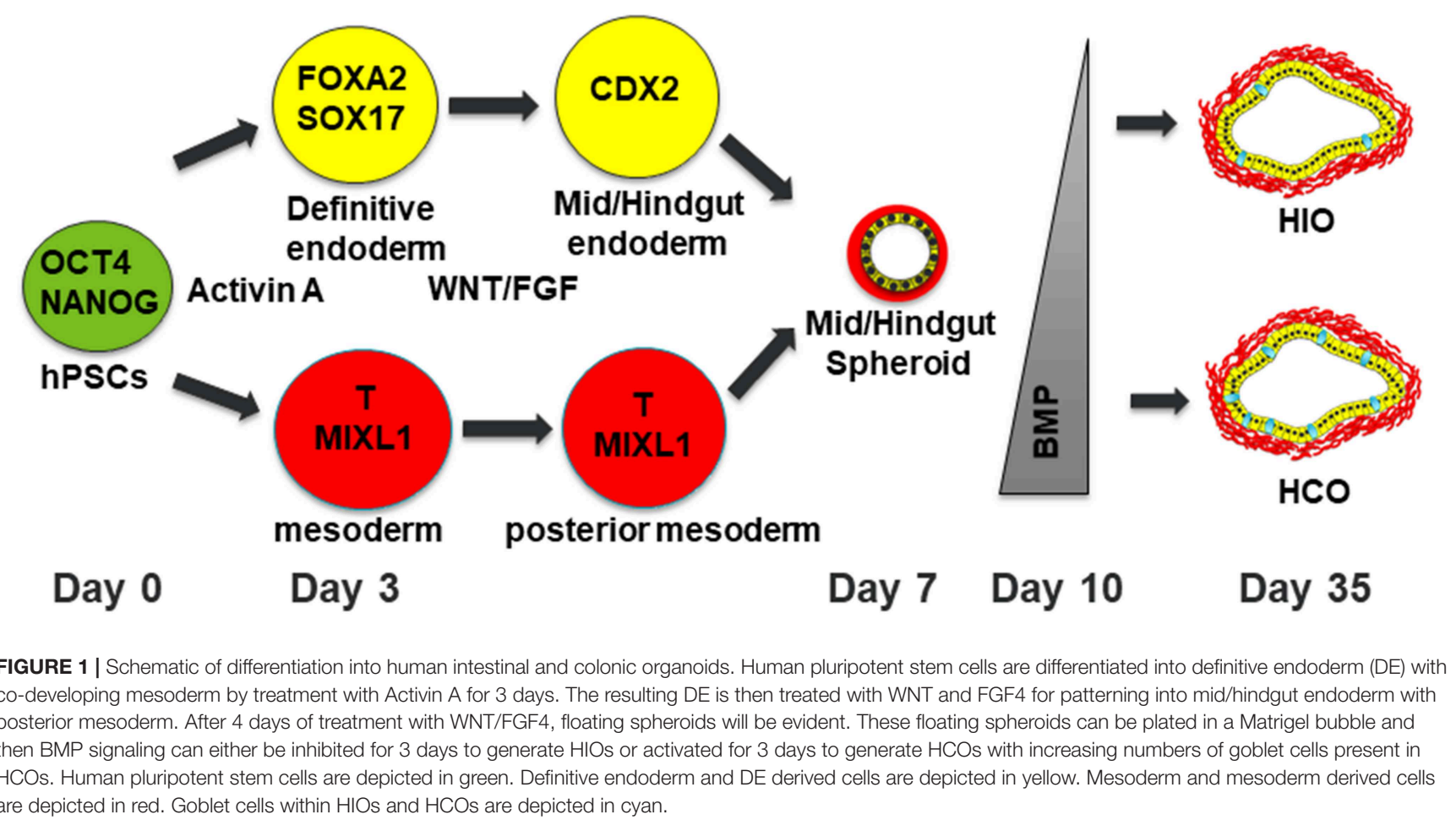

HIOs grown in vitro lack maturity (they lack brush border enzymes, hormone expressing enteroendocrine cells, mature intestinal stem cells, and mature Paneth cells) and thus have limited applications for studying intestinal physiology $(21,22)$. To address this shortcoming, Jung et al. hypothesized that the coculture of HIOs with T lymphocytes (Jurkat cells) would allow the in vitro maturation of these organoids (23). Surprisingly, this co-culture system allowed the expression of maturation markers such as intestinal stem cell expressed olfactomedin 4 (OLFM4), Paneth cell expressed defensin alpha 5 (DEFA5), brush border expressed sucrase isomaltase (SI), dipeptidyl Peptidase 4 DPP4, lactase (LCT), and enteroendocrine cell expressed gastric inhibitory polypeptide (GIP) in HIOs grown in vitro. The authors were able to identify Interleukin 2 (IL-2) as the crucial factor secreted by Jurkat cells which allowed the maturation of the HIOs in vitro. The transcriptomic profile of co-cultured and IL-2 treated HIOs more closely resembled the human adult small intestine. Functional maturation was also evident in the matured HIOs as assessed by their ability to establish barrier function, transport chemotherapeutic drugs and transport glucose. Additionally, matured HIOs expressed functional cystic fibrosis transmembrane conductance regulator (CFTR) and were capable of swelling in response to forskolin, an assay designed to screen for Cystic Fibrosis therapeutic drugs $(24,25)$. The group postulated that IL2 -induced maturation of HIOs could substitute the in vivo maturation and provide an opportunity for large-scale organoid generation, gene editing and drug screening. Although these results were intriguing, it should be noted that HIOs are able to mature following renal transplantation into NOD scid gamma (NSG) mice which lack lymphocytes and have normal intestinal development.

\section{REGIONAL PATTERNING OF HIOs}

Initial analysis of HIOs revealed that they were comprised of proximal and distal small intestinal tissue even within the same organoid suggesting additional signaling might be required for proper patterning of HIOs (8). In order to pattern organoids into region specific segments, two different groups took different approaches. The first group extended the mid-hindgut induction step to show that prolonged WNT and FGF4 signaling could promote the development of distal small intestine (26). This work shed new light on the significance of the dose and length of exposure to growth factors on intestinal regionalization. While short activation of WNT and FGF4 patterned the intestinal organoids into a developing duodenum following renal transplantation, prolonged stimulation led to the development of an intestinal tissue with decreased PDX1, GATA4, ONECUT2, and DMBT1 expression and higher SATB2, GUACA2A, MUC2, and FABP6 levels suggesting an ileal identity.

In another study, Múnera et al. demonstrated that a transient activation of BMP signaling was sufficient to induce the expression of posterior Homeobox (HOX) genes and confer a colonic identity on human gut tube cultures (7). Furthermore, the authors demonstrated that patterning was induced in both the epithelium and mesenchyme of human colonic organoids (HCOs). The resulting HCOs expressed colon enriched HOX genes and special AT-Rich sequence-binding protein 2 (SATB2), a chromatin modifying nuclear protein. In addition, following 


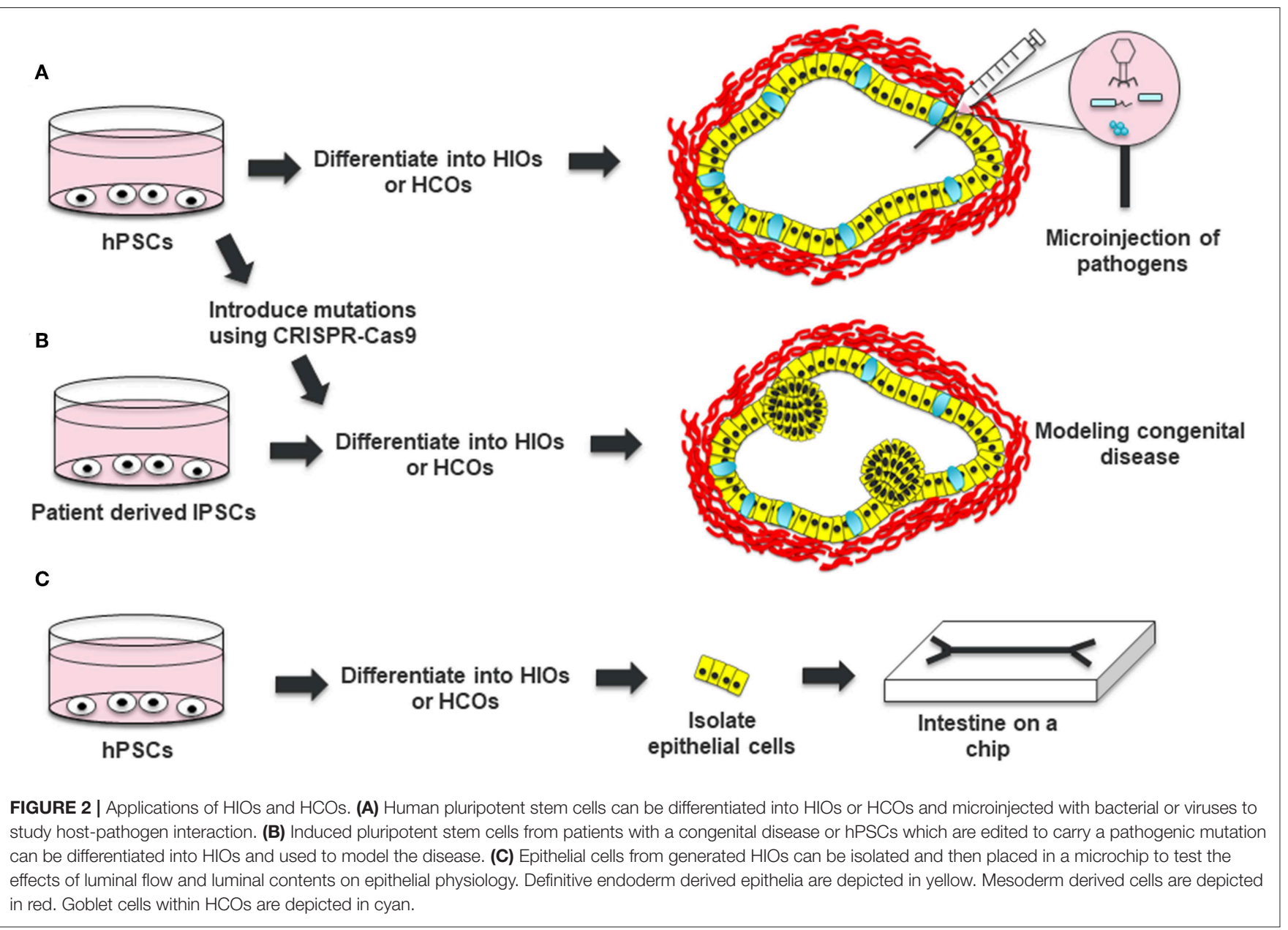

prolonged in vitro culture, HCOs generated colon enriched cell types such as mucin 5B (MUC5B) expressing goblet cells and the colon specific enteroendocrine hormone insulin like 5 (INSL5) following induced expression of NEUROG3. After renal transplantation into immunocompromised mice, HCOs retained the expression of SATB2, lacked GATA4 expression and lacked small intestinal cell types such as GIP expressing enteroendocrine cells and Paneth cells. In contrast, transplanted HIOs expressed GATA4 and contained small intestinal cell types such as Paneth cells and small intestinal enteroendocrine hormones such as Ghrelin, Motilin, and GIP. These studies demonstrated the utility of hPSC-derived organoids for studying mechanisms of anteriorposterior patterning.

\section{MODELING OF INFECTIOUS DISEASE USING HIOs}

Enteric viruses such as rotaviruses and noroviruses are the main infectious agents which cause gastroenteritis and are a primary cause of infant mortality in developing countries (27). Despite their global disease burden, rotaviruses and noroviruses have been difficult to study because suitable in vitro models for viral replication are scarce. HIOs have offered some hope as they have been shown to allow limited replication of clinical isolates of rotaviruses and noroviruses $(22,28)$. The limited replication of these viruses may be due to the fetal nature of HIOs and efforts to improve the in vitro maturation may directly benefit research on enteric viruses. Thus, organoid technology may offer a unique opportunity for future development of vaccines and therapeutics agents for enteric viruses.

HIOs have also been extensively used to study bacterial pathogens including Salmonella enterica Serovar Typhimurium, Shiga toxin (Stx) producing Escherichia coli O157:H7, and Clostridium difficile. Bacterial pathogens can be microinjected into the lumen of HIOs to study the effect of the pathogens on the epithelium and mesenchyme of HIOs. These studies demonstrated that HIOs recapitulate responses to bacterial pathogens including internalization of bacteria, increased secretion of mucus, epithelial barrier disruption and induced pro-inflammatory cytokine expression (29-33). Lees et al. also showed that IL22 can protect the epithelium of HIOs from Salmonella infection (17). In addition, Karve et al. demonstrated that cytokines expressed by HIOs could recruit co-cultured neutrophils into the infected lumen of the organoid (29). This suggests that HIOs combined with immune cells could be an 
attractive model for studying interactions between pathogens, epithelia and immune cells (Figure 2A).

\section{GENETIC TOOLS IN HIO RESEARCH}

HIOs can be used as a tool for studying gene function in the intestine (Figure 2B). In the original work by Spence et al., the authors used adenovirus and lentivirus to study the gain-offunction and loss-of-function of NEUROG3, respectively (8). Adenovirus mediated overexpression of NEUROG3 resulted in an increased number of enteroendocrine cells in HIOs while knockdown of NEUROG3 using lentivirus expressing short hairpin RNAs (shRNAs) resulted in a decreased number of enteroendocrine cells (EECs). Expanding on this work, McCracken an co-workers developed a NEUROG3-inducible system to promote the generation of EECs in gastrointestinal organoids (34). This system has been instrumental to studying the expression of regionally expressed enteroendocrine hormones $(7,34,35)$. In addition, this system has been used to interrogate the functionality of EECs, including the regulation of satiety and hunger inducing hormones in response to luminal nutrients (36).

CRISPR-Cas9 gene editing has also been applied to hPSCs and hPSC-derived HIOs. NEUROG3 deficient hPSCs were previously developed to interrogate the role of NEUROG3 in the development of pancreatic endocrine progenitors (37). Capitalizing on this NEUROG3 null system and the NEUROG3inducible system, Zhang et al. utilized these tools to study the physiology of mutant forms of NEUROG3 on the development of endocrine cells in the pancreas and intestine (38). The authors were able to systematically interrogate the biochemical and functional properties of six different NEUROG3 mutations including those related to protein stability, dimerization and DNA binding. CRISPR-Cas9 gene editing has also been used to study genes associated with Hirschsprung's disease, a disease in which the large intestine is not properly innervated (21). By introducing a mutation in $\mathrm{PHOX} 2 \mathrm{~B}$, researchers were able to demonstrate that PHOX2B deficient NCCs were impaired in their development into cells of the ENS and this resulted in impaired smooth muscle differentiation and in vivo growth of HIOs. CDX2 deficient hPSCs have also been generated and these cells were used to demonstrate the role of this transcription factor in the specification of mid/hindgut vs. foregut fate (39).

The ability to reprogram adult somatic cells into induced pluripotent stem cells (iPSCs) allows the generation of patient specific iPSCs (40). Using this reprogramming approach, Woo et al. generated iPSCs from patients with Dyskeratosis Congenita (DC), a disease which results in shortened telomeres leading to stem cell exhaustion in highly proliferative tissues (41). By using CRISPR-Cas9 gene editing they were able to correct the genetic defect in patient derived cells. Additionally, they demonstrated that WNT signaling was suboptimal in HIOs derived from DC patient iPSCs and that this could be compensated by the addition of a WNT agonist which resulted in increased telomerase activity, telomere length and telomere capping. Additionally, two different groups generated iPSCs from patients with Familial adenomatous polyposis (FAP) $(16,42)$. Both groups were able to demonstrate that organoids grown from FAP patient-derived iPSCs exhibited increased WNT signaling compared to controls. To minimize genetic background effects, the group from Boston University used Talen mediated gene editing to correct the mutant copy of the Adenomatous Polyposis Coli (APC) gene to examine differences between FAP and FAP-corrected HIOs. In contrast, the group from Cornell used normal donors as controls but adjusted organoid growth conditions to generate HCOs. They then used FAP patient derived HCOs to screen for drugs which could restore the expression of APC protein to levels seen in wildtype HCOs. Taken together, these studies demonstrate the utility of HIOs and HCOs for modeling human congenital diseases and for drug screening.

\section{BIOENGINEERING AND HIOs}

The generation of HIOs can be an inefficient process which could limit their scalability. To address this shortcoming, Arora et al. investigated the impact of spheroid number, size and morphology on organoid yield and found the last two parameters to be the key features that help identify spheroids which are most likely to generate organoids (43). By developing an image assisted automated micropipette aspiration and release system, the team was able to isolate "pre-organoids" which led to a significant increase in organoid formation. This study highlights how using a bioengineering approach to select spheroids for plating can increase organoid yields.

The use of Matrigel as a substrate for $\mathrm{HIO}$ growth raises concerns since Matrigel contains the non-human sialic acid Neu5Gc which humans have antibodies against. Thus, alternatives to Matrigel will be required for transplantation of organoids in humans. Synthetic gels could provide a replacement to animal derived extracellular matrices. Cruz-Acuna et al. used a synthetic poly-ethylene glycol-based hydrogel to demonstrate that hydrogels could support the growth of HIOs in vitro. In addition, the authors were able to demonstrate that HIOs grown in this hydrogel could be orthotopically transplanted and could improve mucosal wound healing in the colon of immunocompromised mice (44). A similar study by Capeling et al. demonstrated that Alginate gels could also support the growth and differentiation of HIOs in vitro and in vivo following renal transplantation into immunocompromised mice (45). These studies have provided proof-of-concept that synthetic matrices can be used for HIO growth and transplantation.

HIOs form an internal lumen which hinders the ability to study the effects of bacteria or other factors on the luminal surface of the epithelium. Although protocols have been developed that allow growth of human enteroids and colonoids as monolayers allowing luminal access, these protocols have not been adapted for HIOs $(46,47)$. To address the problem of luminal access in HIOs, Workman et al. isolated epithelial cells from HIOs and grew them in microchips (Figure 2C) (48). This allowed the researchers to optimize the speed of luminal flow necessary for the proper polarization and differentiation of the intestinal epithelial cells. The researchers were able to 
use these intestinal cells on a microchip to study epithelial barrier function in response to luminally administered cytokines. Platforms like this should allow for high throughput analysis of barrier function in response to luminal signals and also help integrate other cell types present in the intestine such as immune cells.

Transplanted HIOs grow as spherical structures with a closed lumen even following transplantation into immunocompromised mice. Developing tissue for transplantation will require the development of tube-like organoids that can be engrafted and that they will maintain luminal flow. In order to generate more tube-like intestinal tissue from HIOs, Poling et al. implanted uniaxial springs into the lumens of HIOs to examine the effect of mechanical stretching on HIO physiology (49). Besides allowing the generation of tube-like HIOs, the mechanical forces applied by the spring allowed an enhanced in vivo maturation of transplanted HIOs based on morphometric analysis, global transcriptional analysis, and functional assays. Moreover, the device increased the tone of the smooth muscles generated by the HIOs. This study highlights how mechanical force can improve organoid morphology and maturation and should aid attempts to transplant such tissue.

\section{FUTURE DIRECTIONS}

HIOs hold tremendous promise for studying the role of epithelial, mesenchymal and neuronal cell types in intestinal physiology and disease. However, immune cells are still absent in HIOs cultures. Multiple immune cell types are present in human intestine including various macrophage subtypes, dendritic cells

\section{REFERENCES}

1. Date S, Sato T. Mini-gut organoids: reconstitution of the stem cell niche. Annu Rev Cell Dev Biol. (2015) 31:269-89. doi: 10.1146/annurev-cellbio-100814-125218

2. Fujii $M$, Clevers $H$, Sato T. Modeling human digestive diseases with CRISPR-Cas9-modified organoids. Gastroenterology. (2019) 156:562-76. doi: 10.1053/j.gastro.2018.11.048

3. Matano M, Date S, Shimokawa M, Takano A, Fujii M, Ohta Y, et al. Modeling colorectal cancer using CRISPR-Cas9-mediated engineering of human intestinal organoids. Nat Med. (2015) 21:256-62. doi: 10.1038/nm. 3802

4. Sato T, Stange DE, Ferrante M, Vries RG, Van Es JH, Van den Brink S, et al. Long-term expansion of epithelial organoids from human colon, adenoma, adenocarcinoma, and Barrett's epithelium. Gastroenterology. (2011) 141:176272. doi: 10.1053/j.gastro.2011.07.050

5. Stelzner M, Helmrath M, Dunn JC, Henning SJ, Houchen CW, Kuo $\mathrm{C}$, et al. A nomenclature for intestinal in vitro cultures. Am J Physiol Gastrointest Liver Physiol. (2012) 302:G1359-63. doi: 10.1152/ajpgi.0049 3.2011

6. Middendorp S, Schneeberger K, Wiegerinck CL, Mokry M, Akkerman RD, van Wijngaarden S, et al. Adult stem cells in the small intestine are intrinsically programmed with their location-specific function. Stem Cells. (2014) 32:108391. doi: 10.1002/stem.1655

7. Múnera JO, Sundaram N, Rankin SA, Hill D, Watson C, Mahe $\mathrm{M}$, et al. Differentiation of human pluripotent stem cells into colonic organoids via transient activation of BMP signaling. Cell Stem Cell. (2017) 21:51-64.e6. doi: 10.1016/j.stem.2017. 05.020 and lymphocytes (50). The use of developmentally inspired methods for generating and co-culturing immune cells should allow for unprecedented studies examining the interaction of immune cells with the various cell types that are present in HIOs. This will allow for improved models of complex diseases such as inflammatory bowel disease (IBD) and intestinal cancers. It should also allow for the interrogation of the developmental origins of gut associated lymphoid tissues (GALT).

The use of microinjection to study bacterial interaction with the epithelium of HIOs is laborious and low-throughput. Recently, Williamson et al. developed a high-throughput platform to microinject biopsy derived organoids (also referred to as colonoids) (51). This platform also allows for automated imaging through visualization of organoids over time. Adapting this platform for use with HIOs and HCOs should allow highthroughput analysis of bacterial pathogens and microbiota.

Although morphologically HIOs undergo transitions similar to those that occur in mammalian embryos, the molecular changes associated with these transitions remain unresolved. Future experiments directly comparing HIOs to human fetal and postnatal intestine from several stages of development will determine the relative age of an $\mathrm{HIO}$ at any timepoint during the in vitro culture. In addition, single cell RNA sequencing of HIOs compared to fetal intestine should allow the identification of cell types which are differentially absent or present in HIOs compared to human fetal intestine.

\section{AUTHOR CONTRIBUTIONS}

$\mathrm{AD}$ and JM helped to write and edit the manuscript.

8. Spence JR, Mayhew CN, Rankin SA, Kuhar MF, Vallance JE, Tolle K, et al. Directed differentiation of human pluripotent stem cells into intestinal tissue in vitro. Nature. (2011) 470:105-9. doi: 10.1038/nature09691

9. Zorn AM, Wells JM. Vertebrate endoderm development and organ formation. Annu Rev Cell Dev Biol. (2009) 25:221-51. doi: 10.1146/annurev.cellbio.042308.113344

10. D'Amour KA, Agulnick AD, Eliazer S, Kelly OG, Kroon E, Baetge EE. Efficient differentiation of human embryonic stem cells to definitive endoderm. Nat Biotechnol. (2005) 23:1534-41. doi: 10.1038/nbt1163

11. Lowe LA, Yamada S, Kuehn MR. Genetic dissection of nodal function in patterning the mouse embryo. Development. (2001) 128:1831-43.

12. Conlon FL, Lyons KM, Takaesu N, Barth KS, Kispert A, Herrmann B, et al. A primary requirement for nodal in the formation and maintenance of the primitive streak in the mouse. Development. (1994) 120:1919-28.

13. Sato T, Vries RG, Snippert HJ, van de Wetering M, Barker N, Stange $\mathrm{DE}$, et al. Single Lgr5 stem cells build crypt-villus structures in vitro without a mesenchymal niche. Nature. (2009) 459:262-5. doi: 10.1038/nature 07935

14. Fordham RP, Yui S, Hannan NR, Soendergaard C, Madgwick A, Schweiger PJ, et al. Transplantation of expanded fetal intestinal progenitors contributes to colon regeneration after injury. Cell Stem Cell. (2013) 13:73444. doi: 10.1016/j.stem.2013.09.015

15. Tamminen K, Balboa D, Toivonen S, Pakarinen MP, Wiener Z, Alitalo $\mathrm{K}$, et al. Intestinal commitment and maturation of human pluripotent stem cells is independent of exogenous FGF4 and Rspondin1. PLoS ONE. (2015) 10:e0134551. doi: 10.1371/journal.pone.01 34551

16. Crespo M, Vilar E, Tsai SY, Chang K, Amin S, Srinivasan T, et al. Colonic organoids derived from human induced pluripotent stem cells 
for modeling colorectal cancer and drug testing. Nat Med. (2017) 23:87884. doi: 10.1038/nm.4355

17. Lees EA, Forbester JL, Forrest S, Kane L, Goulding D, Dougan G. Using human induced pluripotent stem cell-derived intestinal organoids to study and modify epithelial cell protection against salmonella and other pathogens. J Vis Exp. (2019) 147:e59478. doi: 10.3791/ 59478

18. Hill DR, Huang S, Nagy MS, Yadagiri VK, Fields C, Mukherjee $\mathrm{D}$, et al. Bacterial colonization stimulates a complex physiological response in the immature human intestinal epithelium. Elife. (2017) 6:e29132. doi: 10.7554/eLife.29132.031

19. Watson CL, Mahe MM, Munera J, Howell JC, Sundaram N, Poling HM, et al. An in vivo model of human small intestine using pluripotent stem cells. Nat Med. (2014) 20:1310-4. doi: 10.1038/nm.3737

20. Farin HF, Van Es JH, Clevers H. Redundant sources of Wnt regulate intestinal stem cells and promote formation of Paneth cells. Gastroenterology. (2012) 143:1518-29.e7. doi: 10.1053/j.gastro.2012. 08.031

21. Workman MJ, Mahe MM, Trisno S, Poling HM, Watson CL, Sundaram $\mathrm{N}$, et al. Engineered human pluripotent-stem-cell-derived intestinal tissues with a functional enteric nervous system. Nat Med. (2017) 23:4959. doi: $10.1038 / \mathrm{nm} .4233$

22. Finkbeiner SR, Zeng XL, Utama B, Atmar RL, Shroyer NF, Estes MK. Stem cell-derived human intestinal organoids as an infection model for rotaviruses. MBio. (2012) 3:e00159-12. doi: 10.1128/mBio.00 159-12

23. Jung KB, Lee $\mathrm{H}$, Son $\mathrm{YS}$, Lee MO, Kim YD, Oh SJ, et al. Interleukin2 induces the in vitro maturation of human pluripotent stem cell-derived intestinal organoids. Nat Commun. (2018) 9:3039. doi: 10.1038/s41467-018-0 5450-8

24. Dekkers JF, Wiegerinck CL, de Jonge HR, Bronsveld I, Janssens HM, de Winter-de Groot KM, et al. A functional CFTR assay using primary cystic fibrosis intestinal organoids. Nat Med. (2013) 19:939-45. doi: 10.1038/nm.3201

25. Schwank G, Koo BK, Sasselli V, Dekkers JF, Heo I, Demircan T, et al. Functional repair of CFTR by CRISPR/Cas9 in intestinal stem cell organoids of cystic fibrosis patients. Cell Stem Cell. (2013) 13:6538. doi: 10.1016/j.stem.2013.11.002

26. Tsai YH, Nattiv R, Dedhia PH, Nagy MS, Chin AM, Thomson M, et al. In vitro patterning of pluripotent stem cell-derived intestine recapitulates in vivo human development. Development. (2017) 144:104555. doi: 10.1242/dev.138453

27. Girard MP, Steele D, Chaignat CL, Kieny MP. A review of vaccine research and development: human enteric infections. Vaccine. (2006) 24:273250. doi: 10.1016/j.vaccine.2005.10.014

28. Zhang D, Tan M, Zhong W, Xia M, Huang $\mathrm{P}$, Jiang X. Human intestinal organoids express histo-blood group antigens, bind norovirus VLPs, and support limited norovirus replication. Sci Rep. (2017) 7:12621. doi: 10.1038/s41598-017-12736-2

29. Karve SS, Pradhan S, Ward DV, Weiss AA. Intestinal organoids model human responses to infection by commensal and Shiga toxin producing Escherichia coli. PLoS ONE. (2017) 12:e0178966. doi: 10.1371/journal.pone.01 78966

30. Forbester JL, Goulding D, Vallier L, Hannan N, Hale C, Pickard D, et al. Interaction of Salmonella enterica Serovar Typhimurium with intestinal organoids derived from human induced pluripotent stem cells. Infect Immun. (2015) 83:2926-34. doi: 10.1128/IAI.00161-15

31. Leslie JL, Huang S, Opp JS, Nagy MS, Kobayashi M, Young VB, et al. Persistence and toxin production by Clostridium difficile within human intestinal organoids result in disruption of epithelial paracellular barrier function. Infect Immun. (2015) 83:138-45. doi: 10.1128/IAI.02 561-14

32. Engevik MA, Engevik KA, Yacyshyn MB, Wang J, Hassett DJ, Darien B, et al. Human Clostridium difficile infection: inhibition of NHE3 and microbiota profile. Am $J$ Physiol Gastrointest Liver Physiol. (2015) 308:G497-509. doi: 10.1152/ajpgi.0009 0.2014
33. Engevik MA, Yacyshyn MB, Engevik KA, Wang J, Darien B, Hassett DJ, et al. Human Clostridium difficile infection: altered mucus production and composition. Am J Physiol Gastrointest Liver Physiol. (2015) 308:G51024. doi: 10.1152/ajpgi.00091.2014

34. McCracken KW, Cata EM, Crawford CM, Sinagoga KL, Schumacher $\mathrm{M}$, Rockich BE, et al. Modelling human development and disease in pluripotent stem-cell-derived gastric organoids. Nature. (2014) 516:4004. doi: 10.1038/nature13863

35. McCracken KW, Aihara E, Martin B, Crawford CM, Broda T, Treguier $\mathrm{J}$, et al. Wnt/beta-catenin promotes gastric fundus specification in mice and humans. Nature. (2017) 541:182-7. doi: 10.1038/nature 21021

36. Sinagoga KL, McCauley HA, Munera JO, Reynolds NA, Enriquez JR, Watson C, et al. Deriving functional human enteroendocrine cells from pluripotent stem cells. Development. (2018) 145:dev165795. doi: 10.1242/dev.1 65795

37. McGrath PS, Watson CL, Ingram C, Helmrath MA, Wells JM. The basic helix-loop-helix transcription factor NEUROG3 is required for development of the human endocrine pancreas. Diabetes. (2015) 64:2497-505. doi: 10.2337/db14-1412

38. Zhang X, McGrath PS, Salomone J, Rahal M, McCauley HA, Schweitzer J, et al. A comprehensive structure-function study of neurogenin3 disease-causing alleles during human pancreas and intestinal organoid development. Dev Cell. (2019) 50:367-80.e7. doi: 10.1016/j.devcel.2019. 05.017

39. Kumar N, Tsai YH, Chen L, Zhou A, Banerjee KK, Saxena M, et al. The lineage-specific transcription factor CDX2 navigates dynamic chromatin to control distinct stages of intestine development. Development. (2019) 146:dev172189. doi: 10.1242/dev.172189

40. Takahashi K, Tanabe K, Ohnuki M, Narita M, Ichisaka T, Tomoda K, et al. Induction of pluripotent stem cells from adult human fibroblasts by defined factors. Cell. (2007) 131:861-72. doi: 10.1016/j.cell.2007. 11.019

41. Woo DH, Chen Q, Yang TL, Glineburg MR, Hoge C, Leu NA, et al. Enhancing a Wnt-telomere feedback loop restores intestinal stem cell function in a human organotypic model of Dyskeratosis Congenita. Cell Stem Cell. (2016) 19:397-405. doi: 10.1016/j.stem.2016.05.024

42. Sommer CA, Capilla A, Molina-Estevez FJ, Gianotti-Sommer A, Skvir N, Caballero I, et al. Modeling APC mutagenesis and familial adenomatous polyposis using human iPS cells. PLoS ONE. (2018) 13:e0200657. doi: 10.1371/journal.pone.02 00657

43. Arora N, Imran Alsous J, Guggenheim JW, Mak M, Munera J, Wells JM, et al. A process engineering approach to increase organoid yield. Development. (2017) 144:1128-36. doi: 10.1242/dev.142919

44. Cruz-Acuna R, Quiros M, Huang S, Siuda D, Spence JR, Nusrat A, et al. PEG-4MAL hydrogels for human organoid generation, culture, and in vivo delivery. Nat Protoc. (2018) 13:2102-19. doi: 10.1038/s41596-0180036-3

45. Capeling MM, Czerwinski M, Huang S, Tsai YH, Wu A, Nagy MS, et al. Nonadhesive alginate hydrogels support growth of pluripotent stem cell-derived intestinal organoids. Stem Cell Rep. (2019) 12:381-94. doi: 10.1016/j.stemcr.2018.12.001

46. In J, Foulke-Abel J, Zachos NC, Hansen AM, Kaper JB, Bernstein HD, et al. Enterohemorrhagic Escherichia coli reduce mucus and intermicrovillar bridges in human stem cell-derived colonoids. Cell Mol Gastroenterol Hepatol. (2016) 2:48-62.e3. doi: 10.1016/j.jcmgh.2015. 10.001

47. VanDussen KL, Marinshaw JM, Shaikh N, Miyoshi H, Moon C, Tarr PI, et al. Development of an enhanced human gastrointestinal epithelial culture system to facilitate patient-based assays. Gut. (2015) 64:911-20. doi: 10.1136/gutjnl-2013-306651

48. Workman MJ, Gleeson JP, Troisi EJ, Estrada HQ, Kerns SJ, Hinojosa $\mathrm{CD}$, et al. Enhanced utilization of induced pluripotent stem cell-derived human intestinal organoids using microengineered chips. Cell Mol Gastroenterol Hepatol. (2018) 5:669-77.e2. doi: 10.1016/j.jcmgh.2017. 12.008 
49. Poling HM, Wu D, Brown N, Baker M, Hausfeld TA, Huynh $\mathrm{N}$, et al. Mechanically induced development and maturation of human intestinal organoids in vivo. Nat Biomed Eng. (2018) 2:429-42. doi: 10.1038/s41551-018-0243-9

50. Mowat AM, Agace WW. Regional specialization within the intestinal immune system. Nat Rev Immunol. (2014) 14:667-85. doi: 10.1038/nri3738

51. Williamson IA, Arnold JW, Samsa LA, Gaynor L, DiSalvo M, Cocchiaro JL, et al. A high-throughput organoid microinjection platform to study gastrointestinal microbiota and luminal physiology. Cell Mol Gastroenterol Hepatol. (2018) 6:301-19. doi: 10.1016/j.jcmgh.2018.05.004
Conflict of Interest: The authors declare that the research was conducted in the absence of any commercial or financial relationships that could be construed as a potential conflict of interest.

Copyright (C) 2019 Daoud and Múnera. This is an open-access article distributed under the terms of the Creative Commons Attribution License (CC BY). The use, distribution or reproduction in other forums is permitted, provided the original author(s) and the copyright owner(s) are credited and that the original publication in this journal is cited, in accordance with accepted academic practice. No use, distribution or reproduction is permitted which does not comply with these terms. 